Research Articles: Behavioral/Cognitive

\title{
The brain selectively tunes to unfamiliar voices during sleep
}

https://doi.org/10.1523/JNEUROSCI.2524-20.2021

Cite as: J. Neurosci 2022; 10.1523/JNEUROSCI.2524-20.2021

Received: 16 September 2020

Revised: 14 October 2021

Accepted: 5 November 2021

This Early Release article has been peer-reviewed and accepted, but has not been through the composition and copyediting processes. The final version may differ slightly in style or formatting and will contain links to any extended data.

Alerts: Sign up at www.jneurosci.org/alerts to receive customized email alerts when the fully formatted version of this article is published.

Copyright (? 2022 Ameen et al.

This is an open-access article distributed under the terms of the Creative Commons Attribution 4.0 International license, which permits unrestricted use, distribution and reproduction in any medium provided that the original work is properly attributed. 
1 The brain selectively tunes to unfamiliar voices during sleep.

2 [Abbreviated title: Sleeping brain and unfamiliar voices]

3 Mohamed S. Ameen ${ }^{1,2}$, Dominik PJ Heib ${ }^{1,2, *}$, Christine Blume ${ }^{3,4, *}$, Manuel Schabus ${ }^{1,2,+}$

$4 \quad{ }^{1}$ University of Salzburg, Department of Psychology, Laboratory for Sleep, Cognition and Consciousness

5 Research, Hellbrunner Strasse 34, 5020 Salzburg.

$6{ }^{2}$ University of Salzburg, Centre for Cognitive Neuroscience Salzburg (CCNS), Hellbrunner Strasse 34, 5020

7 Salzburg.

$8{ }^{3}$ Psychiatric Hospital of the University of Basel, Centre for Chronobiology, Wilhelm Klein-Strasse 27, 4002 Basel, 9 Switzerland.

$10{ }^{4}$ University of Basel, Transfaculty Research Platform Molecular and Cognitive Neurosciences, Birmannsgasse 8 , $11 \mathrm{CH}-4055$ Basel, Switzerland.

12 * These authors contributed equally.

13 + Corresponding author, email: manuel.schabus@sbg.ac.at

D The authors declare no conflict of interests.

\section{Acknowledgments}

This project was supported by the Austrian Science Fund (FWF; Y777). MS Ameen is

supported by the FWF Austrian Science Fund (W 1233-B) and the Austrian Academy of

Science (OEAW). CB is supported by a fellowship of the Austrian Science Fund (FWF; J-

4243), a grant from the University of Basel, and funds from the Freiwillige Akademische

Gesellschaft (FAG), the Novartis Foundation for Biological-Medical Research, and the

Psychiatric Hospital of the University of Basel (UPK). The authors would like to thank

Renata del Giudice for her support with data collection, Kerstin Hoedlmoser and Malgorzata

Wislowska for their invaluable input throughout the process. 
$\underline{\text { Abstract }}$

The brain continues to respond selectively to environmental stimuli during sleep. However, the functional role of such responses, and whether they reflect information processing or rather sensory inhibition is not fully understood.

Here, we present 17 human sleepers (14 females) with their own name and two unfamiliar first names, spoken by either a familiar voice (FV) or an unfamiliar voice (UFV), while recording polysomnography during a full night's sleep. We detect K-complexes, sleep spindles, and micro-arousals, and assess event-related, and frequency responses as well as inter-trial phase synchronization to the different stimuli presented during non-rapid eye movement (NREM) sleep.

We show that UFVs evoke more K-complexes and micro-arousals than FVs. When both stimuli evoke a K-complex, we observe larger evoked potentials, more precise time-locking of brain responses in the delta band (1-4 Hz), and stronger activity in the high frequency $(>16 \mathrm{~Hz})$ range, in response to UFVs relative to FVs. Crucially, these differences in brain responses disappear completely when no K-complexes are evoked by the auditory stimuli.

Our findings highlight discrepancies in brain responses to auditory stimuli based on their

relevance to the sleeper and propose a key role for K-complexes in the modulation of sensory processing during sleep. We argue that such content-specific, dynamic reactivity to external sensory information enables the brain to enter a 'sentinel processing mode' in which it engages in the important internal processes that are ongoing during sleep while still maintaining the ability to process vital external sensory information. 
59 Previous research has shown that sensory processing continues during sleep. Here, we

60 studied the capacity of the sleeping brain to extract and process relevant sensory

61 information. We presented sleepers with their own names and unfamiliar names spoken by

62 either a familiar (FV) or an unfamiliar voice (UFV). During non-rapid eye movement (NREM)

63 sleep, UFVs elicited more K-complexes and micro-arousals than FVs. By contrasting stimuli

64 that evoked K-complexes, we demonstrate that UFVs evoked larger, more synchronized brain responses as well as stronger power at high frequencies $(>16 \mathrm{~Hz})$ relative to FVs.

66 These differences in brain responses disappeared when no K-complexes were evoked. Our

67 results suggest a pivotal role for K-complexes in the selective processing of relevant

68 information during NREM sleep.

69 


\section{Introduction}

During sleep, the brain continues to respond to auditory stimuli in a selective fashion (Portas et al., 2000; Andrillon et al., 2016; Blume et al., 2017; 2018). Previous studies have demonstrated that, for instance, the subject's own name (SON) evokes stronger brain responses than other names during sleep (Oswald et al., 1960; Perrin et al., 1999; Pratt et al., 1999). Recently, Blume et al. (2018) showed that during all stages of sleep, brain responses to SON and other unfamiliar names (UNs) did not differ; however, names uttered by an unfamiliar voice (UFV) evoked stronger brain responses as compared to a familiar voice $(\mathrm{FV})$.

The discrepancy in brain responses to different stimuli implies the presence of an initial, presumably low-level, sensory processing during sleep that enables the brain to differentiate between sensory signals (Blume et al., 2018). However, knowledge about the functions of such responses is still lacking. That is, the selective brain responses to specific sounds during sleep might reflect inhibitory processes that protect sleep from disruptions. Conversely, they might indicate further, higher level, processing that ensures the connectedness of the sleeping brain to the surrounding.

In this study, we investigated the purpose of such selective brain responses to sounds presented during non-rapid eye movement (NREM) sleep. We focused on sleep-specific events that have been previously linked to information processing, sensory inhibition, or both. That is, we focused on three cardinal sleep-specific electroencephalography (EEG) events, namely, the K-complex (KC), sleep spindles, and micro-arousals.

KCs are $\sim 1 \mathrm{~Hz}$ oscillations and a hallmark of N2 sleep (Loomis et al., 1938, Colrain, 2005; Halász, 2005). KCs occur either spontaneously or in response to sensory stimuli. Spontaneous KCs appear in the EEG signal as well-defined sharp negative wave followed by a positive component with a total duration of at least $0.5 \mathrm{~s}$ (Rechtschaffen and Kales, 1968; Hori et al., 2001). Following sensory perturbation, KCs appear to have two main components; a sharp negative deflection at $\sim 550 \mathrm{~ms}$ (N550) followed by a longer-lasting 
positive wave at 900ms (P900) (Bastien \& Campbell, 1992, Cote et al., 1999; Colrain, 2005; Halász, 2005). Some studies have considered an early positive peak that appears around 200ms (P200, Laurino et al., 2014; 2019) and another negative peak around 350ms (N350, Bastien \& Campbell, 1992, Cote et al., 1999) to be parts of the KC, albeit these components can occur without a KC being elicited. Relevant stimuli have higher propensity to trigger KCs (Halász, 2005). Recent theories suggest that KCs can serve both sleepprotecting as well as arousal-inducing processes (Halász, 2005; Jahnke et al., 2012; Forget et al., 2014; Laurino et al., 2014; Blume et al., 2017; 2018; Legendre et al., 2019; Latreille et al., 2020).

Sleep spindles are also characteristic of N2 sleep. Spindles are thalamocortical oscillations of $11-15 \mathrm{~Hz}$ that last around 0.5 to 2s (De Gennaro and Ferrara, 2003; Fernandez and Lüthi, 2020) that can be triggered by sensory stimuli (Antony and Paller, 2017). They have been repeatedly shown to inhibit sensory processing during sleep (McCormick \& Bal, 1994, Schabus et al., 2012; Blume et al., 2018; Fernandez and Lüthi, 2020). However, recent work challenges this notion (Sela et al., 2016) and even associates spindles with the processing of memory-related sounds presented during NREM sleep (Cairney et al., 2018). Finally, micro-arousals are abrupt shifts in the EEG signal towards theta, alpha and/or high beta (>16 Hz) frequencies (Halász et al., 1979; American Sleep Disorders Association, 1992; Halász et al., 2004) that appear in all sleep stages and are considered windows of information processing during sleep (dos Santos Lima et al., 2019; Halász et al., 2004; Halász, 2005). Micro-arousals are usually preceded by KCs (Colrain 2005; Halász, 2005), yet they have been shown to be correlated with a lower incidence of sleep spindles in the preceding 10s of EEG signal (Ehrhart et al., 1981).

Here, we re-analysed the dataset used in Blume et al. (2018) who recorded polysomnography while presenting SONs and two UNs spoken by either a FV or an UFV during a whole night of sleep. We detected KCs, spindles, and micro-arousals in response 
to these sounds during NREM sleep and hypothesized that the selective auditory-evoked responses support the extraction and processing of relevant sensory information.

\section{Materials and methods}

2.1. Participants: We recruited 20 healthy participants with no reported history of neurological or psychological problems as well as no reported sleep disorders. However, one participant dropped out after the adaptation night and we had to exclude two participants due to technical problems during EEG acquisition. Therefore, we performed the analyses we report here on 17 participants ( 14 females) with a median age of $22.6 \pm 2.3$ years. Before beginning the experiment, all participants signed written informed consents. The experiment was approved by the ethics committee of the University of Salzburg.

2.2. Experimental design: Before the start of the experiment, participants were advised to maintain a regular sleep/wake cycle ( $>\mathrm{h}$ of sleep) for at least four days which we monitored via actigraphy (Fig. 1A). Subsequently, participants spent two nights in the sleep laboratory of the University of Salzburg. The first night was an adaptation night, during which we recorded polysomnography (PSG) data with no auditory stimulation. The second night was an experimental night, during which we recorded PSG data while presenting sounds via loudspeakers throughout the night. In both nights, participants were tested during wakefulness before and after sleep. Briefly, the wakefulness testing consisted of two sessions, a passive-listening session and an active-listening session. Passive listening entails that the participants had to listen to the repeatedly presented auditory stimuli; while active listening means that, they had to count the number of presentations of one specific stimulus chosen by the experimenters. Before the wakefulness testing, participants were stimulated with either a bright (blue-enriched) light or an inactive (sham) light for one hour. The order of the light-stimulation conditions was counterbalanced between the adaptation and the experimental nights across participants. However, the light condition is irrelevant to this study, as we grouped our data over both light conditions. For the purpose of this paper, we will focus primarily on the sleep part of the experimental night. For more details on the 
wakefulness part of the experiment, please refer to Blume et al. (2018). During the experimental night, participants went to bed around their habitual bedtime (8:30pm 11:30pm). Time in bed (TIB) was approximately 8 hours. After 8 hours TiB, we waited for light NREM or REM sleep before waking up the participants (median sleep duration $=480 \pm$ 2.5 minutes). The auditory stimulation started directly after participants went to bed and continued throughout the whole night. We presented auditory stimuli continuously for 90 minutes (Stimulation periods) then paused the presentation for 30 minutes (No-stimulation periods) to allow for periods of undisturbed sleep. This resulted in a 120-minute cycle that we repeated four times throughout the night (Fig. 1B).

2.3. Stimuli: We presented six different auditory stimuli (Fig. 1C) that we personalized for each participant. The stimuli were the subject's own name (SON) and two unfamiliar names (thereafter referred to as UNs) spoken by either a familiar voice (FV) or an unfamiliar voice (UFV). A FV was the voice of someone close to the participant, for example one of the parents. An UFV was the voice of someone unknown to the participant. We did not control for the sex of the voices, but they were matched, that is, the familiar and unfamiliar voices were always both either male or female. We chose UNs that matched the SON in the number of syllables and the frequency of occurrence in the population. The volume for stimulus presentation was adjusted individually for each participant so that the participant could clearly hear the stimulus and still be able to fall asleep. Each stimulus was presented 690 times and the mean duration was $808 \mathrm{~ms} \pm 110 \mathrm{~ms}$. Stimuli were presented in a pseudo-random order and no stimulus was presented twice in a row. The inter-stimulus intervals during sleep were jittered between $2800 \mathrm{~ms}$ to $7800 \mathrm{~ms}$ in $500 \mathrm{~ms}$ steps. Stimulus pre-processing, i.e. denoising and normalization, was done using Audacity@ software (http://audacityteam.org/). Stimulus delivery was controlled by Matlab® (Mathworks, Natick, USA). 
2.4. Brain Data Acquisition: We recorded ongoing brain activity using a high-density EEG 256-channel GSN HydroCel Geodesic Sensor Net (Electrical 478 Geodesics Inc., Eugene, Oregon, USA) and a Net Amps 400 amplifier. The PSG recordings included two electrooculography (EOG) and two chin electromyography (EMG) channels. Data were acquired at a sampling rate of $250 \mathrm{~Hz}$ and $\mathrm{Cz}$ served as the online reference.

2.5 Sleep staging: Sleep staging was performed on 30s epochs using the computerassisted sleep classification system developed by the SIESTA group (Somnolyzer $24 \times 7$; The SIESTA Group Schlafanalyse GmbH, Vienna, Austria; Anderer et al., 2005; Anderer et al., 2010) following the standard criteria recommended by the American Association for Sleep Medicine (AASM, American Academy of Sleep Medicine \& Iber, 2007). We have previously shown that level of agreement between this algorithm and expert human scorers is similar to the level of agreement between human experts (Ameen et al., 2019).

\subsection{The detection of sleep microstructures:}

2.6.1. KC detection: We detected KCs automatically with a wavelet-detection algorithm that was developed by the SIESTA group (The SIESTA Group Schlafanalyse GmbH., Vienna, Austria). The development and validation procedures have been described in detail in Parapatics et al. (2015) and Schwarz et al. (2017), respectively. Briefly, 12 experienced human scorers visually scored KCs in 873 10-minute-epochs of PSG recordings from 189 control subjects and 90 patients. The features of the visually scored KCs were used to set the criteria for detection as well as to create a template $\mathrm{KC}$ that worked as a gold standard for the automatic detection. The detection itself is a two-step process; first, the algorithm detects possible KCs via an approach that combines a matched-filtering detection method and a slow-wave detection method (Woertz et al., 2004). Accordingly, the detection criteria for possible KCs were: 1) a minimum negative-to-positive peak-to-peak amplitude of $50 \mu \mathrm{v}$, and 2) a duration between $480 \mathrm{~ms}$ and $1500 \mathrm{~ms}$. Second, all possible KCs are matched to the prototypical KC template via wavelet analysis and the results are submitted to a linear discriminant analysis (LDA) to select only "real" KCs. For our analysis, we considered real 
KCs to be events that have an LDA score (how likely a specific EEG segment is a KC) of 0.8 or higher. This LDA score corresponds to $61.87 \% \pm 9.14$ of all detected KCs and a mean correlation to the template of $0.87 \pm 0.007$ over all subjects and is a compromise between reliable detections and a sufficient number of detected events for our analyses. Note that an LDA score of 1.7 reflects $98 \%$ detection specificity. Before running the detection algorithm, raw data were down-sampled to $128 \mathrm{~Hz}$ and re-referenced to the contralateral mastoid. We detected $\mathrm{KCs}$ at $\mathrm{C} 3$ and $\mathrm{C} 4$. We only report results from $\mathrm{C} 3$ as the detections were similar between C3 and C4. We only considered events that occurred during N2 and N3 sleep and fulfilled the standard criteria for KC detection (Rechtschaffen and Kales, 1968; Hori et al., 2001). For N3 detections, however, we applied a more strict amplitude criterion as we only selected events with a peak-to-peak amplitude of $75 \mu \mathrm{v}$ or higher (Cote et al., 1999; Nir et al., 2011). We marked the start of KC events as the point of the negative-going zero-crossing of the signal prior to the negative peak. We defined evoked KCs as those events which occurred (started) in the $2000 \mathrm{~ms}$ post-stimulus-onset window. Fig. 2 demonstrates the LDA distribution of the detected KCs as well as some examples of the detected events.

\section{[Please insert Figure 2 about here]}

2.6.2. Spindle detection: Sleep spindles were detected using an algorithm developed by the SIESTA group (ASK analyser, The Siesta Group Schlafanalyse GmbH., Vienna, Austria, Gruber et al., 2015). First, we filtered the raw data between 11 and $16 \mathrm{~Hz}$ and then detected spindle events at frontal $(F 3, F 4, F z)$ and central leads $(C 3, \mathrm{C} 4 \& \mathrm{Cz})$ channels rereferenced to the average of mastoids. We used the criteria described in Schimicek et al. (1994). Only events with an amplitude $>12 \mu v$ and duration between $500 \mathrm{~ms}$ and $2000 \mathrm{~ms}$ were considered. Further validation of the detected spindles was done using LDA in which the detected spindles were compared to a template that is generated based on the visual scoring of sleep spindles in 8730 minutes of PSG data from 189 healthy participants and 90 participants with sleep disorders. For our analyses, we only considered events that occurred during N2 and N3 sleep with an LDA score of 1.7 or higher (for more details see: Anderer et 
al., 2005). In order to identify the frequency of each spindle event, the algorithm preforms period-amplitude analysis of the band-pass filtered signal in the time domain. We subdivided spindles into slow $(11-13 \mathrm{~Hz})$ and fast $(13-15 \mathrm{~Hz})$ spindles based on the dichotomy in their topography and functions (Schabus et al., 2007). We report results from fast spindles detected at C3 and slow spindles detected at F3.

2.6.3. Micro-arousal detection: We detected micro-arousals semi-automatically using an algorithm developed by the SIESTA group (The Siesta Group Schlafanalyse GmbH., Vienna, Austria), and has been described in details in (Anderer et al., 2010). Briefly, the algorithm was developed using the scoring of 12 PSG recordings by six independent experts. It incorporates information from central and occipital channels. First, the algorithm compares the absolute and relative power of nine frequency bands including theta, alpha and high beta $(>16 \mathrm{~Hz}$ ) frequencies between a 3-second test window and a moving ten-second baseline via a series of LDA separately for each channel. Second, the start and end of each event are determined by combining the posterior probabilities of all channels so that the number of micro-arousals per total sleep time is the same for both automatic and visual detections. Although, EMG increases are not necessary for the identification of micro-arousals that occur during NREM sleep, some of the detected micro-arousals showed a concurrent increase in the amplitude of the EMG signal; however, this increase in EMG activity was not time-locked to the high-frequency shifts of the EEG signal. The algorithm detects micro-arousals in all sleep stages, however, for the purpose of this study; we selected micro-arousals that occurred during N2 and N3 only (for examples of the detected micro-arousals see: Fig. 3).

\section{[Please insert Figure 3 about here]}

\subsubsection{The detection of transient microstates (micro-measures of alertness): The} detection of sleep microstates provides a more fine-grained scoring that detects transient changes in sleep architecture in 4 s epochs rather than 30 s, using an algorithm described in Jagannathan et al. (2018). The algorithm uses the Hori scale (Tanaka et al., 1996) to classify the epochs into either awake, drowsy (N1), or asleep (N2) based on the signal from a subset 
of 14 electrodes distributed over frontal, central, parietal, occipital and temporal regions. We extracted stimuli from N2 sleep only and removed stimuli with inter-stimulus intervals of less than $4000 \mathrm{~ms}$. Then, we filtered the data between 0.1 and $30 \mathrm{~Hz}$ before running the algorithm on an equal number of epochs in all conditions.

\subsection{EEG pre-processing and analyses:}

2.7.1. Pre-processing: We performed all the pre-processing steps in EEGLAB v14.1.1b (Delorme \& Makeig, 2004). First, we excluded face and neck channels and downsampled the raw data from $183 \mathrm{EEG}$ channels to $128 \mathrm{~Hz}$. Then, we filtered the data between 0.1 and $40 \mathrm{~Hz}$ using a Butterworth bandpass filter. We performed bad channels rejection and interpolation as well as re-referencing to an average reference using the PREP pipeline described in Bigdely-Shamlo et al. (2015). Finally, we performed independent component analysis using the Adaptive Mixture Independent Component analysis (AMICA) toolbox and visually detected and discarded eye and muscle artefacts. 2.7.2. Event-related analysis: We epoched the pre-processed data into $3000 \mathrm{~ms}$ trials $(-1000 \mathrm{~ms}$ to $2000 \mathrm{~ms}$ relative to stimulusonset). For each participant, we converted ERPs into percent power change relative to the 500ms pre-stimulus-onset window using the formula: (Data - mean baseline values) / mean baseline values. 2.7.3. Time-frequency analysis: Time-frequency representations (TFRs) were computed over $8000 \mathrm{~ms}$ epochs, $(-4000 \mathrm{~ms}$ to $4000 \mathrm{~ms}$ relative to stimulus onset). We choose relatively long epochs to avoid edge artefacts due to the transformation. We calculated TFRs by applying a 500ms hanning window as taper on frequencies from 0.5 to $30 \mathrm{~Hz}$ in $0.5 \mathrm{~Hz}$ frequency steps and $5 \mathrm{~ms}$ temporal steps. Similar to ERPs, we converted participant-specific TFRs into percent power change relative to the $500 \mathrm{~ms}$ pre-stimulus-onset window. 2.7.4. Inter-trial phase coherence estimate (ITPC): Following time-frequency transformation, we extracted the complex Fourier coefficient for each channel, frequency, and time point in every single trial. Then we computed the phase angles in each trial before finally, averaging the single-trial ITPC values over all trials per subject. We performed all analyses in Fieldtrip (Oostenveld et al., 2011; http://fieldtriptoolbox.org). 


\subsection{Statistical Analyses:}

For all of our analyses, we randomly selected an equal number of events/epochs per condition from N2 and N3. Evoked events were defined as events that are detected by our algorithms in the $2000 \mathrm{~ms}$ post-stimulus-onset window. Due to violations to the assumptions of parametric testing, we applied rank-based non-parametric tests using the nparLD function implemented in the nparLD package available in $\mathrm{R}$ (Ngouchi et al., 2012). We report ANOVA-type statistics (ATS), p-values (alpha $=0.05$, two-sided), as well as effect sizes using relative treatment effects (RTE). Generally, RTEs represent the probability of the values from the whole dataset being smaller than a randomly chosen observation from the respective group. Therefore, RTE values range between zero and one. An RTE value of 0.5 means no effect. The higher the RTE value of one condition the higher the probability that a randomly chosen value from that condition is larger than that randomly drawn from the whole dataset, and vice versa. When applicable, we performed post-hoc tests via the 'NparLD' function with Bonferroni's correction for multiple comparisons. For repeated measures at different time points, we performed non-linear mixed regression via generalized linear mixed models (GLMMs) implemented in the 'glmer' function of the 'Ime4' package in R (Bates et al., 2015). Both KC and micro-arousals were non-normally distributed. For K-complexes, we used a GLMM with a Poisson distribution. For micro-arousals, due to the presence of a notable amount of zero counts, we used the zero-inflated Poisson distribution implemented in the 'pscl' package (Zeileis et al., 2008). We added our subjects as effect with random intercepts and slopes. We report the estimates of the fixed effects $(\hat{B})$ and their standard errors, z-values, and p-values. We performed post-hoc interaction tests using marginal means estimates as implemented in the 'emmeans' package in R with Tukey's correction for multiple comparisons and we report Cohen's d effect sizes.

For the more temporally resolved analysis to compare between the latencies of the detected events, we binned the $2000 \mathrm{~ms}$ post-stimulus intervals into bins of $100 \mathrm{~ms}$, then we calculated the mean of the number of events in each bin for each subject and condition, before finally 
submitting these results to the permutation analysis in Fieldtrip. The choice of the bin-size is a compromise between a meaningful temporal resolution and a sufficient statistical power.

For ERP, TFR, and ITPC analyses, we selected equal numbers of epochs per condition $(66.47 \pm 56.25)$ for each subject. For this analysis, we averaged the signal from six frontal channels (F3, F4, F7, F8, Fcz and Fz), using a time window from -500ms to $2000 \mathrm{~ms}$ relative to stimulus onset. We calculated the grand average over all subjects in each condition before submitting the results to the non-parametric cluster-based permutation analysis in fieldtrip (Maris and Oostenveld, 2007). We performed two-sided paired-sample t-tests followed by Monte Carlo's approximation with 5000 permutations (cluster-alpha $=0.05$ and critical alpha $=0.025)$. We report the sum of the t-values $(\Sigma t)$, as well as Cohen's $d$ effect sizes calculated over all possible permutations, channels, time points, and frequencies in the cluster.

\section{Results}

\subsection{Auditory stimulation influenced sleep microstructure but not macrostructure.}

First, we assessed the effects of auditory stimulation on sleep macrostructure. We found that auditory stimulation during sleep does not influence sleep macrostructure. That is, we found no change in sleep macrostructure from the adaptation to the experimental night (Fig. 4A). Specifically, we found an effect of Stage $\left(\operatorname{ATS}(2.61)=62.75, p<0.001, R T E_{\text {wake }}=0.24\right.$, $\left.R T E_{N 1}=0.26, R T E_{N 2}=0.79, R T E_{N 3}=0.70, R T E_{R E M}=0.51\right)$, no effect of the night $(A T S(1)=$ $\left.0.04, p=0.84, R T E_{\text {Nostim }}=0.48, R T E_{\text {stim }}=0.52\right)$ and no interaction Night $\mathrm{X}$ Stage $(A T S(2.56)$ $=0.75, p=0.51)$. Similarly, during the experimental night, we found no difference in sleep macrostructure between periods of stimulation and periods of no-stimulation (Fig. 4B). We found a main effects for stage $\left(A T S(2.51)=53.30, p<0.001, R T E_{\text {wake }}=0.28, R T E_{N 1}=0.18\right.$, $\left.R T E_{N 2}=0.82, R T E_{N 3}=0.74, R T E_{R E M}=0.47\right)$. There was no effect of the stimulation $(A T S(1)$ $\left.=3.36, p=0.07, R T E_{A D A P T}=0.5, R T E_{E X P}=0.5\right)$, and no interaction Stimulation X Stage $(\operatorname{ATS}(2.08)=0.68, p=0.52)$. Even when we opted for a more time-resolved analysis of sleep stages based on the Hori scoring system (Jagannathan et al., 2018; Tanaka et al., 1996) that uses 4 s epochs instead of the classical 30s staging. Again, we found a main effect for stage 
$\left(\operatorname{ATS}(1.64)=146.26, p<0.001, R T E_{\text {wake }}=0.28, \operatorname{RTE}_{N 1}=0.18, R T E_{N 2}=0.82, R T E_{N 3}=0.74\right.$, $\left.R_{T E M}=0.47\right)$. However, there was no effect of the stimulation $(A T S(1.69)=3.36, p=0.19$, $\left.R T E_{A D A P T}=0.5, R T E_{E X P}=0.5\right)$, but a significant interaction Stimulation $X$ Stage $(A T S(1.47)=$ 8.17, $p=0.02)$. Post-hoc pairwise test with Bonferroni's correction for multiple comparisons revealed that auditory stimulation resulted in higher number of sleep epochs $(A T S(1)=$ 12.84, $\left.p<0.001, R T E_{\text {stim }}=0.62, R T E_{\text {nostim }}=0.38\right)$ and lower number of drowsy epochs $\left(\operatorname{ATS}(1)=10.88, p<0.001, R T E_{\text {stim }}=0.4, R T E_{\text {nostim }}=0.6\right)$, suggesting even deeper sleep during stimulation periods.

On the level of sleep microstructure, we compared the densities, i.e. the numbers of events per minute of $\mathrm{N} 2$ and N3, of KCs, slow and fast spindles, as well as micro-arousals between stimulation and no-stimulation periods. We show that auditory stimulation significantly increased the densities of KCs (Fig. 4D; $\left(A T S(1)=19.68, p<0.001, R T E_{s t i m}=0.59, R T E_{\text {nostim }}\right.$ $=0.41$ ) and spindles (Fig. 4E; slow: $A T S(1)=19.68, p<0.001, R T E_{s t i m}=0.58, R_{\text {nostim }}=$ 0.42 - fast: $\left.A T S(1)=8, p=0.005, R T E_{s t i m}=0.58, R T E_{\text {nostim }}=0.42\right)$. However, the increase in the density of micro-arousals did not reach statistical significance (Fig. 4F; ATS(1) =1.01, $p=0.31, R T E_{\text {stim }}=0.53, R T E_{\text {nostim }}=0.47$.

\section{[Please insert Figure 4 about here]}

Next, we performed a more temporally resolved analysis of the auditory-induced changes in sleep microstructure. Specifically, we compared the numbers of KCs, spindles, and microarousals between Stimulus-ON and Stimulus-OFF periods during the experimental night. Stimulus-ON periods are $2000 \mathrm{~ms}$ post-stimulus intervals (0 to $2000 \mathrm{~ms}$ relative to stimulusonset), while stimulus-OFF periods are $2000 \mathrm{~ms}$ intervals that start at least $2000 \mathrm{~ms}$ after the onset of the previous stimulus and during which no sounds are presented (Fig. 5A). We found significantly higher numbers of $\mathrm{KCs}\left(A T S(1)=54.77, \mathrm{p}<0.001, \mathrm{RTE}_{\mathrm{ON}}=0.68, \mathrm{RTE}_{\mathrm{OFF}}\right.$ $=0.32)$, slow spindles $\left(A T S(1)=9.13, \mathrm{p}=0.002, \mathrm{RTE}_{\mathrm{ON}}=0.57, R T E_{O F F}=0.43\right)$, fast spindles $\left(A T S(1)=20.16, \mathrm{p}<0.001, R_{T E N}=0.55, R T E_{O F F}=0.45\right)$, and micro-arousals $(A T S(1)=$ 
8.45, $\left.p=0.003, R T E_{O N}=0.58, R T E_{O F F}=0.42\right)$, in the stimulus-ON than in the stimulus-OFF periods (Fig. 5B-E).

\section{[Please insert Figure 5 about here]}

\subsection{The brain responds selectively to unfamiliar voices during NREM sleep.}

We subsequently sought to investigate whether brain responses to auditory stimuli differ depending on their content, i.e. the names and/or voices used in the stimulus. We employed a non-parametric test from the nparLD package with two within factors, i.e. Name (SON and UNs) and Voice (FV and UFV). KC responses to auditory stimuli showed a significant effect of Voice, as UFVs triggered more KCs than FVs (Fig. 6A, ATS(1) $=16.10, p>0.001$, RTE $_{\text {UFV }}$ $\left.=0.76, R T E_{F V}=0.24\right)$, no effect of Name $\left(\operatorname{ATS}(1)=0.09, p=0.76, R_{T E N}=0.48, R_{T E} E_{U N S}=\right.$ $0.52)$, and a significant interaction Name $\times$ Voice $($ ATS $(1)=11.86, p=0.001)$. Post-hoc tests revealed that the amount of KCs triggered by the combination FV-SON was marginally higher than that triggered by the combination FV-UNs $\left(A T S(1)=4.17, p_{\text {bonf }}=0.08, R_{T E} E_{F V S O N}\right.$ $\left.=0.56, R T E_{F V U N s}=0.44\right)$, while there was no difference between UFV-SON and UFV-UNs $\left(A T S(1)=4.17, p_{\text {bonf }}=0.19\right.$, RTE $_{\text {UFVSON }}=0.53$, RTE UFVUNs $\left.=0.47\right)$.

For fast and slow spindles, however, there was no effect of Voice (Fig. 6B-C; slow: $\operatorname{ATS}(1,16)<0.001, p=0.99, \operatorname{RTE}_{U F V}=0.5, \operatorname{RTE}_{F V}=0.5-$ fast: $\operatorname{ATS}(1,16)=2.71, p=0.10$ $\left.R_{T E} E_{U V V}=0.53, R T E_{F V}=0.47\right)$, no effect of Name (slow: $A T S(1,16)=0.46, p=0.5, R_{T E N}=$ $0.51, R_{U N E_{U N}}=0.49$ - fast: $\left.\operatorname{ATS}(1,16)=0.62, p=0.43, R_{T E N}=0.49, \operatorname{RTE}_{U N s}=0.51\right)$, and no interaction Name $X$ Voice (slow: $A T S(1,16)=0.11, p=0.74, R_{\text {UFVSON }}=0.51, R_{\text {TEFVUNS }}$ $=0.49, R_{T E} E_{F S O N}=0.52, R T E_{F V U N S}=0.48$ - fast: $\operatorname{ATS}(1,16)=2.63, p=0.10, R T E_{U F V S O N}=$ $\left.0.50, R T E_{U F V U N S}=0.55, R T E_{F V S O N}=0.47, R T E_{F V U N S}=0.47\right)$. Micro-arousals showed a main effect of Voice (Fig. 6D, ATS(1) $=9.14, p=0.002, R_{T E} E_{U F V}=0.59, R T E_{F V}=0.41$ ), no effect of Name $\left(\operatorname{ATS}(1)=1.16, p=0.29, R_{T E} E_{S O N}=0.53, R T E_{U N S}=0.47\right)$ and no interaction Name $X$ Voice $\left(\operatorname{ATS}(1)=0.19, p=0.66, \operatorname{RTE}_{\text {UFVSON }}=0.61, R_{T E} E_{\text {UFVUNS }}=0.58, R_{T E} E_{F S O N}=0.44\right.$, $\left.R T E_{F V U N S}=0.37\right)$. 
Fig. 6E depicts the number of KCs and micro-arousals evoked by different stimulus types as compared to a $2000 \mathrm{~ms}$ no-stimulation period. Further, a temporally-resolved analysis (see section 2.8) showed that the difference in the evoked KCs between FVs and UFVs occurred in the $100 \mathrm{~ms}-800 \mathrm{~ms}$ post-stimulus window (Fig. $6 \mathrm{~F}, \Sigma t(16)=25.35, p=0.002, d=1.02$ ). For micro-arousals (Fig. 6G), the difference appeared $200 \mathrm{~ms}$ post-stimulus-onset, i.e. $100 \mathrm{~ms}$ later than that of KCs, and was significant between $200 \mathrm{~ms}$ and $700 \mathrm{~ms}$ post-stimulus (200400ms: $t(16)=5.92, p=0.01, d=0.84 ; 500-700 \mathrm{~ms}: t(16)=5.19, p=0.02, d=0.76)$. It is worth mentioning that we observed a transient increase in the number of micro-arousals around $200 \mathrm{~ms}$ before the onset of UFV stimuli, however, this increase did not yield a significant statistical difference.

\section{[Please insert Figure 6 about here]}

To illustrate whether the unfamiliarity of the voice is candidly the main cause of the difference in $\mathrm{KC}$ and micro-arousal responses, we divided the night into halves and hypothesized that brain responses to UFVs, but not to FVs, will decrease from the first to the second half, as the UFVs become more familiar with time. We modelled the change in the number of KCs from the first to the second half of the night using a GLMM with Poisson distribution fit by maximum likelihood (Fig. 7A). We found a main effect of Voice $(\hat{B}=1.17 \pm 0.16, z=7.51, p<$ 0.001), no effect of Time $(\hat{B}=-0.08 \pm 0.11, z=-0.77, p=0.44)$, and a significant interaction Time $X$ Voice $(z=-2.28, p=0.02)$. Post-hoc tests revealed a significant decrease of the UFV-triggered KCs from the first to the second half of the night $(\hat{B}=-0.31 \pm 0.08, z=3.54, p$ $=0.002, d=0.31)$, while FV-evoked KCs did not change $(\hat{B}=-0.08 \pm 0.11, z=0.77, p=0.87$, $d=0.08$ ). Conversely, for micro-arousals, a GLMM with zero-inflated Poisson distribution (Fig. 7B) demonstrated a marginally significant effect of Voice $(\hat{B}=0.89 \pm 0.51, z=1.75, p=$ 0.08), no effect of Time $(\hat{B}=-0.19 \pm 0.27, z=0.73, p=0.98)$, and no interaction Time $X$ Voice $(\hat{B}=-0.09 \pm 0.33, z=-0.27, p=0.55)$. We provide a more detailed description of the GLMMs results in Fig. 7C-D.

[Please insert Figure 7 about here] 


\subsection{UFVs evoke stronger K-complex mediated brain responses during NREM sleep.}

Next, we aimed to examine the neural dynamics underlying the aforementioned differences in sleep microstructure. Therefore, we compared the ERPs between FVs and UFVs in two conditions: 1) when the stimuli triggered KCs, 2) when no KCs were triggered. We found that when KCs were triggered, UFVs evoked a larger, more pronounced negative peak (Fig. 8A, $\left.\sum t(16)=-436.35, p>0.001, d=0.96\right)$ that resembles the KC's N550 in its temporal and morphological characteristics. Importantly, however, we observed that the peak of the N550 potential occurred later ( 750-800ms) than the usual time window $(500-550 \mathrm{~ms})$ and had a much smaller amplitude $(20-50 \mu v)$ than that $(\sim 100 \mu v)$ previously reported in the literature (Colrain, 2005; Halász, 2005; Laurino et al., 2014). We illustrate that these discrepancies with previous literature are due to the relatively large temporal window we defined for the detection of evoked KCs $(2000 \mathrm{~ms})$ as well as our decision to use an average reference as compared to the mastoid reference used in earlier studies (Bastien and Campbell, 1992; 1994; Colrain, 2005; Halász, 2005; Laurino, 2014). Fig. 8-1 and Fig. 8-2 in the extended data show the difference in the amplitude of the N550 according to the different referencing procedures as well as the latency jitter of the N550 peak on the single-trial level. Further, to confirm that the difference in the ERPs does not reflect a difference in the amplitude of the evoked KCs, we compared the peak-to-peak amplitudes of the evoked KCs, as measured by the detection algorithm, between FVs and UFVs (Fig. 8B). Peak-to-peak amplitudes showed no significant effect of Voice $\left(\operatorname{ATS}(1)=1.47, p=0.23, \operatorname{RTE}_{U F V}=0.48, \operatorname{RTE}_{F V}=0.52\right)$, Name $\left(A T S(1)=0.71, p=0.39, R T E_{S O N}=0.52, R T E_{U N S}=0.48\right)$, and no interaction Voice $X$ Name $\left(A T S(1)=1.47, p=0.23, R T E_{U F V S O N}=0.49, R T E_{U F V U N S}=0.47, R T E_{F V S O N}=0.54, R T E_{F V U N S}=\right.$ 0.50). Intriguingly, when no KCs are evoked, we found no difference in the amplitude of the ERPs between UFVs and FVs (Fig. 8C, $0.3-0.37 \mathrm{~s}: \sum \mathrm{t}(16)=27.31, \mathrm{p}=0.13$ ).

[Please insert Figure 8 about here]

\subsection{K-complex mediated brain responses to UFVs reflect sensory processing}


We then speculated that the difference in the amplitude of the ERPs, if not due to a difference in the amplitude of the evoked KCs, might reflect more synchronized evoked responses to UFVs as compared to FVs. Therefore, we compared the phase consistency of brain responses between FVs and UFVs via the inter-trial phase coherence metric (ITPC, Tallon-Baudry et al. 1996). We observed stronger ITPC following UFVs as compared to FVs in the delta band $(1-4 \mathrm{~Hz}$; Fig $9 \mathrm{~A}, \Sigma t(16)=2413.98, p=0.003, d=1.06)$ indicating more synchronized brain responses to UFVs. Fig. 9B shows the ITPC contrast between FVs and UFVs indicating that difference in the IPTC is a product of stronger time-locked responses to UFVs. Similar to our ERP analysis, in the absence of the evoked KCs, IPTC values did not differ between FVs and UFVs (Fig. 9C, $\Sigma t(16)=2521.7, p=0.001$ ).

Finally, we performed spectral analysis of brain responses to UFVs and FVs in the presence and absence of evoked KCs. We observed stronger low frequency response (delta band; 1 $4 \mathrm{~Hz}$ ) to UFVs as compared to FVs (Fig. 9D-E) that started around $250 \mathrm{~ms}$ post stimulus onset. This delta responses appeared independent of the presence (Fig. 9D, $\Sigma t(16)=$ 9395.64, $p<0.001, d=1.25$ ), or absence (Fig. 9E, $\Sigma t(16)=4097.56, p=0.013, d=0.86$ ) of the evoked KC.

Crucially, only in the presence of KCs, UFVs additionally elicited a significant increase in the power of higher frequencies $(>16 \mathrm{~Hz}$ ) as compared to FVs (Fig. 9D, $\Sigma t(16)=5020.62, p=$ $0.006, d=1.04)$ starting around $500 \mathrm{~ms}$ post-stimulus.

\section{[Please insert Figure 9 about here}

\section{Discussion}

In this study, we presented sleepers with their own first names (SON) and two unfamiliar names (UNs) spoken by either a familiar voice (FV) or an unfamiliar voice (UFV) during a full night of sleep with polysomnography. We show that although auditory stimulation did change sleep architecture (Fig. 4A-C); they induced prominent, stimulus-specific changes in sleep microstructure. Generally, presenting auditory stimuli during NREM sleep increased the number of KCs, spindles, and micro-arousals (Fig. 5). UFVs triggered more KCs (Fig. 6A) 
and micro-arousals (Fig. 6D) than FVs. However, we found no difference in the amount of triggered KCs, spindles, or micro-arousals between SONs and UNs. The difference in the numbers of evoked KCs and micro-arousals between FVs and UFVs appeared around $100 \mathrm{~ms}$ post-stimulus (200ms for micro-arousals) and extended over the whole duration of the stimuli $~ 800 \mathrm{~ms}$ (Fig. 6F-G). While the number of the UFV-triggered KCs decreased in the second half of the night, the numbers of evoked micro-arousals remained relatively stable throughout the night (Fig. 7). Moreover, in the presence of the auditory-evoked KC, UFVs triggered larger evoked responses (Fig. 8A) that did not reflect a difference in the amplitudes of the evoked KCs (Fig. 8B-C) but rather more-synchronized brain responses to UFVs relative to FVs (Fig. 9A-B). Similarly, brain responses to UFVs demonstrated an increase in the power of high frequencies $(>16 \mathrm{~Hz}$ ), suggesting a stronger arousal reaction to UFVs (Fig. 9D). Crucially, we were not able to detect such differential brain responses between FVs and UFVs in the absence of the evoked KC.

It has previously been suggested that the more relevant the stimulus, the higher its tendency to trigger KCs (Halász, 2005). In this regard, our results pose UFVs as more relevant - or in evolutionary terms potentially more threatening (Blume et al., 2018) - and consequently more arousing to the sleeper than FVs. Indeed, the increase in micro-arousals following UFVs suggests a transient shift towards external processing of "vital" environmental stimuli. In the same vein, the decrease in the number of UFV-evoked KCs in the second half as compared to the first half of the night (Fig. 7A) supports the notion that the sleeping brain continues to learn new information during sleep (Züst et al., 2019). That is, homeostatic regulatory processes alone cannot explain this observation, since the number of FV-evoked KCs did not change, indicating a stimulus-specific attenuation of brain responses. It might be that the sleeping brain learns, through repeated processing, that an initially unfamiliar stimulus poses no immediate threat to the sleeper and consequently decreases its response to it. Conversely, in a safe sleep environment, the brain might be "expecting" to hear FVs and consistently inhibits any response to such stimuli to preserve sleep (see Fig. 6E). Although this assumption remains speculative, it entails a thorough investigation of the brain's ability to 
generate top-down predictions of the external sensory world during sleep. Nevertheless, our results suggest that the unfamiliarity of voice is a strong promoter of brain responses during NREM sleep.

What is the role of the auditory-evoked brain responses during NREM sleep? Central to such responses is the $\mathrm{KC}$, i.e. the most prominent sleep-specific response to sensory stimulation. To answer this question, we contrasted trials during which FVs and UFVs triggered KCs. In such trials, UFVs evoked a larger negative potential that resembles the N550 component of the KC (Fig. 7A). The N550 has been associated with large-scale neuronal silencing that protects sleep (Cash et al., 2009; Laurino et al., 2014), and conversely, an arousal reaction that facilitates stimulus processing (Atienza et al., 2001). In our study, the amplitudes of the evoked KCs did not differ between FVs and UFVs corroborating previous literature presenting the evoked KC as an all-or-non phenomenon (Bastein and Campbell, 1992). Rather, the difference in the N550-amplitude between FVs and UFVs was due to more synchronized brain responses to UFVs as indicated by the ITPC values (Fig. 9A). Stimulusinduced phase modulations have been suggested to promote information processing and transmission in the cortex (Canavier, 2015; Lakatos et al., 2013; Voloh and Womelsdorf, 2016), and increased ITPC values have been associated with better cognitive performance (Hanslmayr et al., 2005; Eidelman-Rothman et al., 2019) and enhanced attention (Joon Kim et al., 2007) during wakefulness. Together, our results suggest that the preferential brain responses to UFVs during NREM sleep reflect sensory processing. Importantly, the shorter post-stimulus time window during which UFVs elicited KCs as compared to FVs, as indicated by the narrower peak in Fig. 6F, implies better temporal alignment of KCs following UFVs and emphasizes the contribution of the evoked KCs to the observed phase modulations. Finally, the stronger high frequency responses $(>16 \mathrm{~Hz}$, Fig. 9) to UFVs that appeared only in the presence of KCs corroborate the role of the auditory-evoked KCs in promoting sensory processing of relevant information. Together, our findings suggest a central role for KCs in the extraction and processing of relevant external sensory information during NREM sleep. 
We did not find any differences in brain responses between SON and UNs in contrast

to previous literature (Oswald et al., 1960; Perrin et al., 1999). One explanation might be that while sleep preserves low-level auditory processing, it attenuates higher-order linguistic tracking (Makov et al., 2017). We speculate that this is due to the disruption of the activity of the large-scale networks necessary for higher-order name processing (Démonet et al., 1992; Luke et al., 2002), due to the loss of long-range cortical connectivity during sleep (Massimini et al., 2005). Further research should elucidate the mechanisms of language tracking during sleep.

We show that the auditory-evoked spindles are not influenced by specific characteristics of the auditory stimuli (i.e., name or voice). However, the role of spindles in response to sensory stimuli during sleep is far from clear. Previous research has shown spindles attenuate or even inhibit the processing of auditory information (De Gennaro and Ferrera, 2003; Schabus et al., 2012; Blume et al., 2018). Other work suggests that brain responses are preserved during spindles (Sela et al., 2016) and even argues for a role for spindles in the processing of memory-related sensory information (Antony et al. 2018; Cairney et al. 2018; Jegou et al. 2019). More research should investigate the role of spindles in response to sensory information irrelevant to ongoing memory processes.

Finally, we found no relevant changes in sleep macrostructure and architecture due to auditory stimulation (Fig. 4). In fact, micro-arousals represent an integral part of healthy sleep as they ensure its reversibility (Halász et al., 2004). Hence, their slight increase should not be viewed as a disruption to normal sleep. Further, the analysis of microstates even indicates a shift towards deeper sleep in response to UVF stimuli (Fig. 4C), which is most likely a byproduct of more auditory-evoked KCs that influenced the staging. However, the classical 30s sleep staging might not be sensitive enough to capture subtle changes in sleep microstructure. Hence, the development and refinement of new fine-grained methods, such as the Hori-based microstate classification (Jagannathan et al., 2018) promises better monitoring of transient sleep fluctuations, especially in the presence of sensory perturbation. 
In summary, sleep appears to be far from a homogenous state of unconsciousness.

565 There are temporal windows in sleep during which the brain filters, extracts, and processes

566 relevant external information. We speculate that such content-specific, dynamic reactivity to

567 sensory signals enables the brain to enter a "sentinel processing mode" (Blume et al., 2018)

568 during which it preserves the ability to efficiently engage in the important internal processes

569 that are ongoing during sleep while remaining connected to the surrounding environment.

\section{References:}

57Ameen MS, Cheung LM, Hauser T, Hahn MA, Schabus M (2019) About the Accuracy and 572 Problems of Consumer Devices in the Assessment of Sleep. Sensors 19:4160.

57American Association of Sleep Medicine (AASM), Iber C (2007) The AASM manual for the scoring 574 of sleep and associated events: rules, terminology, and specifications.

57Anderer P, Gruber G, Parapatics S, Woertz M, Miazhynskaia T, Klosch G, Saletu B, Zeitlhofer J, 576 Barbanoj MJ, Danker-Hopfe H, Himanen S-L, Kemp B, Penzel T, Grozinger M, Kunz D,

577 Rappelsberger P, Schlogl A, Dorffner G (2005) An E-health solution for automatic sleep 578 classification according to Rechtschaffen and Kales: validation study of the Somnolyzer $24 \mathrm{x}$ 5797 utilizing the Siesta database. Neuropsychobiology 51:115-133.

58Anderer P, Moreau A, Woertz M, Ross M, Gruber G, Parapatics S, Loretz E, Heller E, Schmidt A, 581 Boeck M, Moser D, Kloesch G, Saletu B, Saletu-Zyhlarz GM, Danker-Hopfe H, Zeitlhofer J, 582 Dorffner $\mathrm{G}$ (2010) Computer-assisted sleep classification according to the standard of the 583 American Academy of Sleep Medicine: validation study of the AASM version of the 584 Somnolyzer $24 \times 7$. Neuropsychobiology 62:250-264.

58Andrillon T, Poulsen AT, Hansen LK, Léger D, Kouider S (2016) Neural Markers of 586 Responsiveness to the Environment in Human Sleep. J Neurosci 36:6583-6596.

58Antony JW, Paller KA (2017) Using Oscillating Sounds to Manipulate Sleep Spindles. Sleep 40.

58Antony JW, Piloto L, Wang M, Pacheco P, Norman KA, Paller KA (2018) Sleep Spindle 589 Refractoriness Segregates Periods of Memory Reactivation. Curr Biol 28:1736-1743.e4.

59Association (ASDA) ASD (1992) Arousals scoring rules and examples : a preliminary report from 591 sleep disorders atlas task force of the American Sleep Disorders Association. Sleep 15:173592184.

59Atienza M, Cantero JL, Escera C (2001) Auditory information processing during human sleep as 594 revealed by event-related brain potentials. Clin Neurophysiol 112:2031-2045.

59Bastien C, Campbell K (1992) The evoked K-complex: all-or-none phenomenon? Sleep 15:236596245.

59 Bastien C, Campbell K (1994) Effects of rate of tone-pip stimulation on the evoked K-Complex. 598 Journal of Sleep Research 3:65-72.

59Bates D, Mächler M, Bolker B, Walker S (2015) Fitting Linear Mixed-Effects Models Using Ime4. 600 Journal of Statistical Software 67:1-48. 
60Berry RB, Brooks R, Gamaldo CE, Harding SM, Marcus C, Vaughn BV (2012) The AASM manual 602 for the scoring of sleep and associated events. Rules, Terminology and Technical 603 Specifications, Darien, Illinois, American Academy of Sleep Medicine 176:2012.

60Bigdely-Shamlo N, Mullen T, Kothe C, Su K-M, Robbins KA (2015) The PREP pipeline: 605 standardized preprocessing for large-scale EEG analysis. Front Neuroinform 9:16.

60Blume C, del Giudice R, Lechinger J, Wislowska M, Heib DPJ, Hoedlmoser K, Schabus M (2017) 607 Preferential processing of emotionally and self-relevant stimuli persists in unconscious N2 608 sleep. Brain and Language 167:72-82.

60Blume C, Del Giudice R, Wislowska M, Heib DPJ, Schabus M (2018) Standing sentinel during 610 human sleep: Continued evaluation of environmental stimuli in the absence of 611 consciousness. Neuroimage 178:638-648.

61Cairney SA, Guttesen A á V, Marj NE, Staresina BP (2018) Memory Consolidation Is Linked to 613 Spindle-Mediated Information Processing during Sleep. Current Biology 28:948-954.e4.

61氏anavier CC (2015) Phase-resetting as a tool of information transmission. Curr Opin Neurobiol $615 \quad 31: 206-213$.

616ash SS, Halgren E, Dehghani N, Rossetti AO, Thesen T, Wang C, Devinsky O, Kuzniecky R, 617 Doyle W, Madsen JR, Bromfield E, Erőss L, Halász P, Karmos G, Csercsa R, Wittner L, 618 Ulbert I (2009) The Human K-Complex Represents an Isolated Cortical Down-State. Science $619324: 1084-1087$.

62Colrain IM (2005) The K-complex: a 7-decade history. Sleep 28:255-273.

62 Cote KA, de Lugt DR, Langley SD, Campbell KB (1999) Scalp topography of the auditory evoked 622 K-complex in stage 2 and slow wave sleep. J Sleep Res 8:263-272.

62Be Gennaro L, Ferrara M (2003) Sleep spindles: an overview. Sleep Med Rev 7:423-440.

62Delorme A, Makeig S (2004) EEGLAB: an open source toolbox for analysis of single-trial EEG 625 dynamics including independent component analysis. J Neurosci Methods 134:9-21.

62Démonet JF, Chollet F, Ramsay S, Cardebat D, Nespoulous JL, Wise R, Rascol A, Frackowiak R 627 (1992) The anatomy of phonological and semantic processing in normal subjects. Brain 115 ( 628 Pt 6):1753-1768.

62dos Santos Lima GZ, Lobao-Soares B, Corso G, Belchior H, Lopes SR, de Lima Prado T, 630 Nascimento G, França AC de, Fontenele-Araújo J, Ivanov PC (2019) Hippocampal and 631 cortical communication around micro-arousals in slow-wave sleep. Scientific Reports 9:5876.

63Ehrhart J, Ehrhart M, Muzet A, Schieber JP, Naitoh P (1981) K-complexes and sleep spindles 633 before transient activation during sleep. Sleep 4:400-407.

63任idelman-Rothman M, Ben-Simon E, Freche D, Keil A, Hendler T, Levit-Binnun N (2019) 635 Sleepless and desynchronized: Impaired inter trial phase coherence of steady-state 636 potentials following sleep deprivation. Neurolmage 202:116055.

63Fernandez LMJ, Lüthi A (2020) Sleep Spindles: Mechanisms and Functions. Physiol Rev $638 \quad 100: 805-868$.

63Eorget D, Morin CM, Bastien CH (2011) The Role of the Spontaneous and Evoked K-Complex in 640 Good-Sleeper Controls and in Individuals with Insomnia. Sleep 34:1251-1260.

64t1alász P (2005) K-complex, a reactive EEG graphoelement of NREM sleep: an old chap in a new 642 garment. Sleep Med Rev 9:391-412. 
64Błalász P, Kundra O, Rajna P, Pál I, Vargha M (1979) Micro-arousals during nocturnal sleep. Acta 644 Physiol Acad Sci Hung 54:1-12.

64Elalász P, Terzano M, Parrino L, Bódizs R (2004) The nature of arousal in sleep. J Sleep Res $646 \quad 13: 1-23$.

64AlansImayr S, Klimesch W, Sauseng P, Gruber W, Doppelmayr M, Freunberger R, Pecherstorfer $648 \mathrm{~T}$ (2005) Visual discrimination performance is related to decreased alpha amplitude but 649 increased phase locking. Neuroscience Letters 375:64-68.

65Blori T, Sugita Y, Koga E, Shirakawa S, Inoue K, Uchida S, Kuwahara H, Kousaka M, Kobayashi 651 T, Tsuji Y, Terashima M, Fukuda K, Fukuda N, Sleep Computing Committee of the Japanese 652 Society of Sleep Research Society (2001) Proposed supplements and amendments to "A 653 Manual of Standardized Terminology, Techniques and Scoring System for Sleep Stages of 654 Human Subjects", the Rechtschaffen \& Kales (1968) standard. Psychiatry Clin Neurosci 655 55:305-310.

65ஞagannathan SR, Ezquerro-Nassar A, Jachs B, Pustovaya OV, Bareham CA, Bekinschtein TA 657 (2018) Tracking wakefulness as it fades: Micro-measures of alertness. Neuroimage 658 176:138-151.

659ahnke K, von Wegner F, Morzelewski A, Borisov S, Maischein M, Steinmetz H, Laufs H (2012) 660 To wake or not to wake? The two-sided nature of the human K-complex. Neuroimage 661 59:1631-1638.

66łegou A, Schabus M, Gosseries O, Dahmen B, Albouy G, Desseilles M, Sterpenich V, Phillips C, 663 Maquet P, Grova C, Dang-Vu TT (2019) Cortical reactivations during sleep spindles following 664 declarative learning. Neuroimage 195:104-112.

66500 Kim Y, Grabowecky M, Paller KA, Muthu K, Suzuki S (2007) Attention induces 666 synchronization-based response gain in steady-state visual evoked potentials. Nature 667 Neuroscience 10:117-125.

66Bakatos P, Musacchia G, O'Connell MN, Falchier AY, Javitt DC, Schroeder CE (2013) The 669 spectrotemporal filter mechanism of auditory selective attention. Neuron 77:750-761.

67batreille V, von Ellenrieder N, Peter-Derex L, Dubeau F, Gotman J, Frauscher B (2020) The 671 human K-complex: Insights from combined scalp-intracranial EEG recordings. Neurolmage 672 213:116748.

67Baurino M, Menicucci D, Piarulli A, Mastorci F, Bedini R, Allegrini P, Gemignani A (2014) 674 Disentangling different functional roles of evoked K-complex components: Mapping the 675 sleeping brain while quenching sensory processing. Neuroimage 86:433-445.

676aurino M, Piarulli A, Menicucci D, Gemignani A (2019) Local Gamma Activity During Non-REM 677 Sleep in the Context of Sensory Evoked K-Complexes. Front Neurosci 13 Available at: 678 https://www.ncbi.nlm.nih.gov/pmc/articles/PMC6803494/ [Accessed July 6, 2020].

67Begendre G, Andrillon T, Koroma M, Kouider S (2019) Sleepers track informative speech in a 680 multitalker environment. Nat Hum Behav 3:274-283.

68toomis AL, Harvey EN, Hobart GA (1938) Distribution of disturbance-patterns in the human 682 electroencephalogram with special reference to sleep. Journal of Neurophysiology 1:413683430.

68tuke K-K, Liu H-L, Wai Y-Y, Wan Y-L, Tan LH (2002) Functional anatomy of syntactic and 685 semantic processing in language comprehension. Hum Brain Mapp 16:133-145. 
68\$lakov S, Sharon O, Ding N, Ben-Shachar M, Nir Y, Golumbic EZ (2017) Sleep Disrupts High687 Level Speech Parsing Despite Significant Basic Auditory Processing. J Neurosci 37:7772_ 6887781.

68Maris E, Oostenveld R (2007) Nonparametric statistical testing of EEG- and MEG-data. J 690 Neurosci Methods 164:177-190.

69Massimini M, Ferrarelli F, Huber R, Esser SK, Singh H, Tononi G (2005) Breakdown of cortical 692 effective connectivity during sleep. Science 309:2228-2232.

69B/cCormick DA, Bal T (1994) Sensory gating mechanisms of the thalamus. Current Opinion in 694 Neurobiology 4:550-556.

69Elir Y, Staba RJ, Andrillon T, Vyazovskiy VV, Cirelli C, Fried I, Tononi G (2011) Regional Slow 696 Waves and Spindles in Human Sleep. Neuron 70:153-169.

69\loguchi K, Gel YR, Brunner E, Konietschke F (2012) nparLD: An R Software Package for the 698 Nonparametric Analysis of Longitudinal Data in Factorial Experiments. Journal of Statistical 699 Software 50:1-23.

700ostenveld R, Fries P, Maris E, Schoffelen J-M (2010) FieldTrip: Open Source Software for 701 Advanced Analysis of MEG, EEG, and Invasive Electrophysiological Data Baillet S, ed. 702 Computational Intelligence and Neuroscience 2011:156869.

70Bswald I, Taylor AM, Treisman M (1960) Discriminative responses to stimulation during human 704 sleep. Brain 83:440-453.

70Barapatics S, Anderer P, Gruber G, Saletu B, Saletu-Zyhlarz G, Dorffner G (2015) K-complex 706 amplitude as a marker of sleep homeostasis in obstructive sleep apnea syndrome and 707 healthy controls. Somnologie 19:22-29.

70Berrin F, García-Larrea L, Mauguière F, Bastuji H (1999) A differential brain response to the 709 subject's own name persists during sleep. Clin Neurophysiol 110:2153-2164.

718ortas CM, Krakow K, Allen P, Josephs O, Armony JL, Frith CD (2000) Auditory processing 711 across the sleep-wake cycle: simultaneous EEG and fMRI monitoring in humans. Neuron $712 \quad 28: 991-999$.

71Bratt H, Berlad I, Lavie P (1999) 'Oddball' event-related potentials and information processing 714 during REM and non-REM sleep. Clinical Neurophysiology 110:53-61.

71Bechtschaffen A, Kales A (1968) A Manual of Standardized Terminology, Techniques and Scoring 716 System for Sleep Stages of Human Subjects.

718chabus M, Dang-Vu TT, Albouy G, Balteau E, Boly M, Carrier J, Darsaud A, Degueldre C, 718 Desseilles M, Gais S, Phillips C, Rauchs G, Schnakers C, Sterpenich V, Vandewalle G, 719 Luxen A, Maquet $P(2007)$ Hemodynamic cerebral correlates of sleep spindles during human 720 non-rapid eye movement sleep. Proc Natl Acad Sci USA 104:13164-13169.

72\$chabus M, Dang-Vu TT, Heib DPJ, Boly M, Desseilles M, Vandewalle G, Schmidt C, Albouy G, 722 Darsaud A, Gais S, Degueldre C, Balteau E, Phillips C, Luxen A, Maquet P (2012) The Fate

723 of Incoming Stimuli during NREM Sleep is Determined by Spindles and the Phase of the

724 Slow Oscillation. Front Neurol 3 Available at:

725 https://www.ncbi.nlm.nih.gov/pmc/articles/PMC3319907/ [Accessed April 12, 2020].

726chimicek P, Zeitlhofer J, Anderer P, Saletu B (1994) Automatic sleep-spindle detection 727 procedure: aspects of reliability and validity. Clin Electroencephalogr 25:26-29. 
728chwarz JFA, Åkerstedt T, Lindberg E, Gruber G, Fischer H, Theorell-Haglöw J (2017) Age 729 affects sleep microstructure more than sleep macrostructure. J Sleep Res 26:277-287.

736ela Y, Vyazovskiy VV, Cirelli C, Tononi G, Nir Y (2016) Responses in Rat Core Auditory Cortex 731 are Preserved during Sleep Spindle Oscillations. Sleep 39:1069-1082.

73̄̄allon-Baudry C, Bertrand O, Delpuech C, Pernier J (1996) Stimulus specificity of phase-locked 733 and non-phase-locked $40 \mathrm{~Hz}$ visual responses in human. J Neurosci 16:4240-4249.

734anaka H, Hayashi M, Hori T (1996) Statistical features of hypnagogic EEG measured by a new 735 scoring system. Sleep 19:731-738.

73甘oloh B, Womelsdorf T (2016) A Role of Phase-Resetting in Coordinating Large Scale Neural

737 Networks During Attention and Goal-Directed Behavior. Front Syst Neurosci 10 Available at: 738 https://www.frontiersin.org/articles/10.3389/fnsys.2016.00018/full [Accessed April 6, 2021].

73QVoertz M, Miazhynskaia T, Anderer P, Dorffner G (2004) Automatic K-complex detection: 740 comparison of two different approaches.

$74 \mathbb{Z}$ eileis A, Kleiber C, Jackman S (2008) Regression Models for Count Data in R. Journal of 742 Statistical Software 27:1-25.

74Z̈̈̈st MA, Ruch S, Wiest R, Henke K (2019) Implicit Vocabulary Learning during Sleep Is Bound to 744 Slow-Wave Peaks. Curr Biol 29:541-553.e7.

\section{6. Figure legends:}

746 Figure 1. Experimental design. A) Protocol: participants were invited for an initial screening

747 interview during which they were given the wrist-worn actigraphy and advised to keep a

748 regular sleep-wake cycle. Participants slept in the sleep laboratory during two nights: an

749 adaptation night during which PSG was recorded but no stimuli were presented, and an

experimental night during which we recorded PSG and presented auditory stimuli throughout

751 the night. B) Procedure of the experimental night: participants slept for approximately eight

752 hours with PSG and auditory stimulation. All participants went through a stimulation session in

753 the evening before sleep; however, this session is irrelevant to the current manuscript. The

754 auditory stimuli started directly after going to bed and continued for 90 minutes, then paused

755 for $\sim 30$ minutes to allow for a period of undisturbed sleep. This cycle was repeated four times

756 accounting for the whole duration of the night. C) (Top) Stimuli: We presented the subject's

757 own name (SON) and two unfamiliar names (UN1 \& UN2) spoken by either a familiar voice

758 (FV) or an unfamiliar voice (UFV). (Bottom): an exemplary sequence of stimulus presentation.

759 Stimuli were presented in a pseudo-random order; each stimulus was presented 690 times

760 and the inter-stimulus intervals ranged between $2800 \mathrm{~ms}$ to $7800 \mathrm{~ms}$. 
Figure 2. K-complex detection and examples. A) Linear discriminant analysis (LDA) scores for the all detected K-complexes for all subjects. The dashed line represents our minimum cut-off at an LDA value of 0.8 , which is the threshold used for the selection of reliable K-complex events. The percentage of the events used in our analyses from all the detected events for each subject are indicated in red at the bottom. B) Examples of the detected K-complexes at channel C3 referenced to the contralateral mastoid. We show the standard EEG montage that we used for sleep staging as well as event detections. Specifically, we used channels (from top to bottom): F3, F4, C3, C4, O1, and $\mathrm{O} 2$ referenced to the contralateral mastoid. Moreover, we show one EMG and two EOG channels as well as the average of both mastoids. Examples one and two show K-complexes detected in N2 sleep from one subject, while examples three and four are K-complexes detected during N3 in a different subject.

Figure 3. Examples of the detected micro-arousals. We show the standard EEG montage that is used for sleep staging as well as event detections. Specifically, we used channels (from top to bottom): $\mathrm{F} 3, \mathrm{~F} 4, \mathrm{C} 3, \mathrm{C} 4, \mathrm{O} 1$, and $\mathrm{O} 2$ referenced to the contralateral mastoid. Moreover, we show one EMG and two EOG channels as well as the average of both mastoids. Examples 1 and 2 are events detected in N2 sleep from one subject. Example 2 shows a micro-arousal that is preceded by a K-complex. Example 3 is from an N3 epoch from a different subject.

Figure 4. Auditory stimulation influenced sleep microstructure but not macrostructure. In each participant, we selected equal numbers of epochs for all conditions. A) Difference in sleep architecture, i.e. the distribution of sleep stages, between the adaptation and the experimental night. To account for the effect of sleep-onset latency, we discarded all epochs that preceded the first N1 epoch. We found no difference between the architectures of both nights. B) Difference in sleep architecture between stimulation and no-stimulation periods during the experimental night. The experimental night consisted of periods of continuous auditory stimulation and period of no stimulation. We found no difference in the sleep 
architecture between those periods. C) Microstate differences between the stimulation and the no stimulation periods. To circumvent the poor temporal resolution of classical sleep staging in 30sec epochs, we opted for a more time-resolved analysis of sleep stages based on the Hori scoring system. We found a higher number of sleep epochs and lower number of drowsy epochs during the stimulation periods suggesting deeper sleep during auditory stimulation. D-F) A comparison of the densities of sleep microstructure between stimulation and no-stimulation periods. D) K-complex density during the stimulation periods is higher than that in the no-stimulation periods. E) Slow and Fast spindles densities are higher during the stimulation periods. F) Micro-arousal density, however, did not differ between stimulation and no-stimulation periods. Boxplots show the median and the whiskers depict the $25 \%$ and the $75 \%$ quartiles. Each dot/triangle represents one participant in one condition. ${ }^{*} p<0.05$, ${ }^{* *} p<0.001$.

\section{Figure 5. Auditory stimulation increased the occurrence sleep microstructures. A) A} graphical illustration of the stimulus-ON and stimulus-OFF windows used for the analysis. We detected events in the 2000 ms post-stimulus window (Stimulus-ON; green), as well as during $2000 \mathrm{~ms}$ pre-stimulus windows that are at least $2000 \mathrm{~ms}$ after the last stimulus (Stimulus-OFF, orange). The numbers of (B) K-complexes, (C) slow spindles, (D) fast spindles, as well as (E) micro-arousals were significantly higher during the stimulus-ON than the stimulus-OFF periods. Y-axis depicts the percentage of epochs in which events were detected. Boxplots show the median and the whiskers depict the $25 \%$ and the $75 \%$ quartiles. Each dot/triangle represents one participant in one condition. ${ }^{*} p<0.05,{ }^{* *} p<0.001$.

Figure 6. Selective sleep-specific responses to unfamiliar voices during NREM sleep. A) Differences in the triggered K-complexes between familiar voices (FV) and unfamiliar voices (UFV) in the $2000 \mathrm{~ms}$ post-stimulus-onset window. UFVs triggered more K-complexes than FVs. However, the numbers of (B) slow and (C) fast spindles did not differ between FV and UFVs. D) Differences in triggered micro-arousals between FVs and UFVs demonstrating the higher number of micro-arousals triggered by UFVs. E) The amount of triggered K- 
complexes and micro-arousals by all of our stimulus categories and compared to the same number of $2000 \mathrm{~ms}$ no-stimulation epochs. F-G) Temporal aspects of the difference in the triggered K-complexes and micro-arousals. F) The difference between UFV and FV in the number of triggered K-complexes was significant from $100 \mathrm{~ms}$ to $800 \mathrm{~ms}$ (indicated by bar and asterisks). G) The difference in the number of micro-arousals between FVs and UFVs was significant in the periods from 200 to $400 \mathrm{~ms}$, and from 500 to $700 \mathrm{~ms}$. Boxplots show the median and the whiskers depict the $25 \%$ and the $75 \%$ quartiles. Each dot/triangle represents one participant in one condition. In panels $F$ and $G$ The dashed horizontal line on top of the plots represent the mean duration of our stimuli $(808 \mathrm{~ms})$, the lines depicts the means of the bins, and the shadings show the standard error of the mean. ${ }^{*} \mathrm{p}<0.05,{ }^{* *} \mathrm{p}<0.001$, SONs: Subject's own name, UNs: Unfamiliar names. FVSON: familiar voice speaking the subject's own name. UFVSON: unfamiliar voice speaking the subject's own name, FVUNs: familiar voice speaking two unfamiliar names, and UFVUNs: unfamiliar voice speaking two unfamiliar names.

\section{Figure 7. The effect of time on sleep-specific responses to unfamiliar voices during} NREM sleep. A) The difference in the numbers of triggered K-complexes to familiar voices (FV) and unfamiliar voices (UFV) from the first to the second half of the night. A GLMM using Poisson distribution revealed a significant interaction Time $x$ Voice, as only the amount of UFV-triggered K-complexes decreased in the second half. B) The number of triggered microarousals did not change from the first and the second half of the night. C) A statistical report of the GLM model of K-complexes. We added Time (first half \& second half), and Voices (familiar and unfamiliar voices) as fixed effects. Moreover, we assigned random intercepts and slopes for each subject. We found a significant main effect of voice, no effect of time, and a significant interaction Time $X$ Voice. D) Statistical report of the zero-inflated Poisson GLM of micro-arousals. Similar to K-complexes, we added Time (first half \& second half), and Voices (familiar and unfamiliar voices) as fixed effects and random intercepts and slopes for each subject. No main effect of time and no interaction Time $x$ Voice, indicating that the amount of triggered micro-arousals did not change from the first to the second half of the 
night. " $p<0.05$, SONs: Subject's own name, UNs: Unfamiliar names. Boxplots show the median and the whiskers depict the $25 \%$ and the $75 \%$ quartiles. Each dot/triangle represents one participant in one condition

\section{Figure 8. Unfamiliar voices elicited stronger brain responses in the presence of the} evoked K-complex. A) ERP contrast between familiar voice (FV) and unfamiliar voices (UFV) stimuli in the presence of the auditory-evoked K-complexes. UFVs triggered a larger amplitude of the evoked response between $510 \mathrm{~ms}$ to $1400 \mathrm{~ms}$ as shown by the grey shadings. Figures 8-1 and 8-2 illustrate the methodological underpinnings of the discrepancies between the amplitude and latency of the negative component in panel $A$ as compared to the conventional amplitude and latency of the N550 in the literature. B) Comparison of the peak-to-peak amplitude (P2P; N550 to P900) of the evoked K-complexes showing no difference in the amplitudes of the evoked K-complexes between FVs and UFVs. C) ERP responses to FVs and UFVs in the absence of evoked K-complexes. There was no difference in the ERP amplitudes between FVs and UFVs when no K-complexes were evoked. The solid blue and red lines and the shadings represent the mean and the standard error of the mean respectively. The dashed horizontal line on top of the plots represent the mean duration of our stimuli $(808 \mathrm{~ms})$. Vertical dashed lines (at $\mathrm{x}=0$ ) represent stimulus onset. The red dots on the topographical plots at the bottom-left side of the plots indicate the locations of the channels used for the analysis. Boxplots show the median and the whiskers depict the $25 \%$ and the $75 \%$ quartiles. Each dot/triangle represents one participant in one condition.

Figure 8-1. K-complex trials referenced to contralateral mastoids. In the upper row, we show the comparison between the evoked K-complexes to FVs and UFVs centered on the start of the K-complex, i.e. negative-going zero-crossing of the K-complex. In more details, we extracted the K-complexes that were detected by our algorithm in the $2000 \mathrm{~ms}$ poststimulus window and created epochs from $-0.5 \mathrm{~s}$ to $2 \mathrm{~s}$ around the start of the K-complex events (negative going zero-crossing). The bottom row depicts the trials where a K-complex 
was evoked, centered on the start of the stimulus (zero = stimulus onset). We show the grand average ERP over all subjects (left panel). Note that the amplitude of the negative wave exceeds 100uv and that there is no difference in the amplitude of the ERPs between FVs and UFVs. We also show the single subject data of the K-complex averaged over trials for each subject (middle panel). Finally, we show single trials ( $n=20$ trials per condition) from one subject (right panel). Together, this figure corroborates our claim that the difference in the ERPs between FVs and UFVs shown in Fig. 8A of the manuscript is due to difference in time-locking rather than the amplitude of the evoked K-complexes. K-complexes were detected at C3. We show data from one electrode F3, referenced to the contralateral mastoid (A2). Shadings reflect the standard error of the mean. Vertical dashed lines (at $x=0)$ represent the start of the evoked K-complex detected by our algorithm (top) or stimulus onset (bottom). Shadings represent the standard error of the mean.

Figure 8-2. K-complex trials referenced to an average reference. Similar to Fig. 8-1, in the upper row we show the evoked K-complexes to FV and UFV centered on start of the Kcomplexes (the zero-crossing of the signal from positive to negative), while the bottom row depicts the trials when a K-complex was evoked centered around the start of the stimuli. The difference between Fig. 8-1 and Fig 8-2 is the choice of the reference. Note the decrease in the amplitude of the ERPs from Fig 8-1 to Fig 8-2 due to changing the reference (from a mastoid reference in Fig. 8-1 to an average reference in Fig. 8-2). Left panel: Grand average ERPs over all subjects for both conditions FVs and UFVs. Middle panel: single subject data for the K-complex trials averaged over all trials. Right panel: Single trial data $(n=20$ per condition) from one subject. K-complexes were detected at C3. We show ERPs from one electrode (F3) referenced to an average reference. Vertical dashed lines (at $\mathrm{X}=0$ ) represent the start of the K-complex event (top) or stimulus onset (bottom). Shadings represent the standard error of the mean.

Figure 9. Brain responses to unfamiliar voices in the presence of the evoked Kcomplex reflect further processing. A) The difference in ITPC values between familiar 
897 voice (FV) and unfamiliar voice (UFV) stimuli in the presence of the evoked K-complex.

898 UFVs evoked significantly higher ITPC than FV in the delta $(1-4 \mathrm{~Hz})$ frequency band. Note

899 that the largest difference in phase locking overlaps with the difference between the ERPs in

900 Fig. 8A. B) Separate ITPC plots for Familiar voices (top) and unfamiliar voices (bottom)

901 showing stronger ITPC values following UFVs and indicating that the difference in ITPC

902 values is due to an increase in ITPC following UFVs. C) ITPC difference between UFVs and

903 FVs in the absence of the evoked K-complexes. There is no difference in ITPC between FVs

904 and UFVs in the absence of evoked K-complexes. D-E) Spectral power maps of the power

905 differences between FVs and UFVs. We demonstrate stronger responses to UFV in a broad

906 frequency range $(\sim 1-10 \mathrm{~Hz})$ regardless of the presence $(D)$ or the absence $(E)$ of K-

907 complexes. However, stronger high frequency $(>16 \mathrm{~Hz})$ responses to UFVs appeared only in

908 the presence of K-complexes. The dashed horizontal line on top of the plots represent the

909 mean duration of our stimuli $(808 \mathrm{~ms})$. Vertical dashed lines (at $\mathrm{x}=0$ ) represent stimulus

910 onset. The red dots on the topographical plots at the bottom-left side of the plots indicate the

911 locations of the channels used for the analysis. 


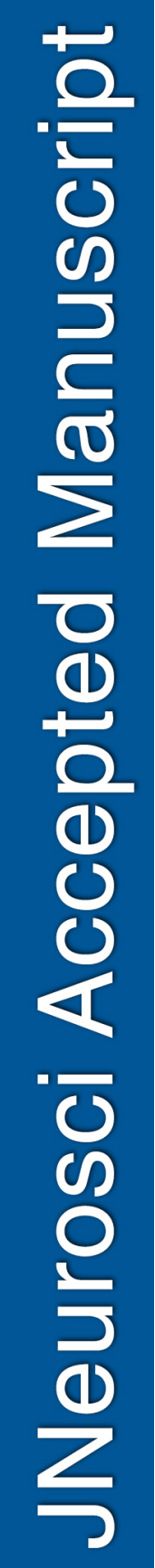


